\title{
An Efficient Heterogeneous Signcryption Scheme from Certificateless to Identity-based Cryptosystem
}

\author{
Shufen Niu ${ }^{1}$, Zhenbin $\mathrm{Li}^{1,{ }^{*}}$, Miao Tian ${ }^{1}$, Caifen Wang ${ }^{1}$ and Xiangdong Jia ${ }^{1}$ \\ ${ }^{1}$ College of Northwest Normal University of Computer Science and Engineering, Lanzhou, 730070, PR China
}

\begin{abstract}
The heterogeneous signcryption can not only realize the confidentiality and un-forgeability of the information transmission between different public key cryptography environments, but also reduce the communication cost. The proposed scheme uses bilin-ear pairings between certificateless cryptography and identity-based cryptography to construct a heterogeneous signcryption scheme. In addition, two cryptography systems use different secret master keys. Furthermore, the proposed scheme has the indistinguishability against adaptive chosen ciphertext attacks and existential unforgeability against adaptive chosen-message attacks in the random oracle model. Through the data analysis, this scheme is more effective than other similar types of scheme.
\end{abstract}

Keywords. Heterogeneous signcryption, Certificateless cryptography, Identity-based cryptography, Different secret master, Secure

\section{Introduction}

Data confidentiality and authentication should be ensured when transmitting data in public networks. The traditional approach, signature then encryption involves numerous calculations, costly communication, and low computational efficiency. To improve the computational efficiency, Zheng[1] proposed the notion of signcryption in 1997. Signcryption can simultaneously perform signature and encryption, which enhances the efficiency of communication systems.

In the future, heterogeneous network technology is crucial to secure communication. Generally, different cryptosystems should be used in heterogeneous networks. Heterogeneous Signcryption (HSC) should be explored to ensure the confidentiality and authentication of messages in a heterogeneous network.

In 2010, Sun[2]. proposed the HSC scheme that communicates from Traditional Public Key Instruction (TPKC) to Identity-based Public Key Cryptography (IDPKC)[3]. However, the scheme only addresses external security threats[4]. In 2011, Huang et al.[5] proposed a HSC scheme with key privacy, which satisfies internal security[4]. In 2013, $\mathrm{Fu}$ et al.[6] proposed an IDPKC-to-TPKC construction of a multireceiver signcryption scheme. In the same year, Li et al. [7] proposed a two-way HSC scheme. However, these HSC schemes only consider the signcryption problem between IDPKC and TPKC. Although these schemes use different public key cryptosystems, the sender and the receiver use the same system parameters. In 2016, Zhang et al.[8] proposed a HSC scheme that communicates from Certificateless Public Key Cryptography (CLPKC)
$[9,10]$ to TPKC. To the best of our knowledge, only four HSC schemes have been developed, and the signcryption problem between IDPKC and CLPKC has not been addressed in the literature.

Existing heterogeneous signcryption schemes use similar master secret keys. In 2016, Li et al.[11] proposed a multi-receiver signcryption scheme for heterogeneous systems. Their work provided inspiration for the current study.

Based on $\mathrm{Li}[12]$, the proposed scheme develops a scheme from CLPKC[13] to IDPKC. The present paper introduces a formal security model called Efficient CLPKC-to-IDPKC Heterogeneous Signcryption Scheme and proves the model's semantic security and existential unforgeability in the random oracle model [14]. Our scheme is more secure and efficient than other existing schemes. The proposed scheme has the following features.

- This scheme uses different master secret keys in different cryptosystem systems, and improve the security of the system.

- Compared with existing heterogeneous schemes, this scheme is reduced system uptime through reducing the number of bilinear pairs. This scheme is more secure and efficient.

- In the random oracle model, this scheme ensures the confidentiality and un forge ability of data in the context of the Variants Decisional Bilinear Dif-fie-Hellman Problem (VDBDHP), Variants Computational Bilinear Dif-fie-Hellman Problem (VCBDHP) and Discrete Logarithm Problem (DLP).

The rest of this paper is organized as follows. Related knowledge are reviewed in Section 2.The formal 
definition and security model of the proposed scheme are described in Section 3. The heterogeneous signcryption scheme is presented in Section 4. The security analysis of the scheme is discussed in Section 5. The performance analysis is discussed in Section 6. Finally, Section 7 concludes the paper.

\section{Related knowledge}

This section briefly describe bilinear maps and hard problems.

Let $G_{1}$ be a cyclic additive group generated by $P$, with a prime order $\mathrm{q}$, and $G_{2}$ be a multiplicative group of the same order. The bilinear pairing is a map $e: G_{1} \times G_{1} \rightarrow G_{2}$ with the following properties.

-Bilinearity: $e(a P, b Q)=e(P, Q)^{a b}$ for all $P, Q \in G_{1}$ and $a, b \in Z_{q}^{*}$.

- Non-degeneracy: $P, Q \in G_{1}$ such that $e(P, Q) \neq 1$.

- Computability: An efficient algorithm is available for computing $e(P, Q)$ for any $P, Q \in G_{1}$.

The hard problems involved in the security of the proposed scheme are described as follows.

\subsection{Hard Problems}

- Variants Decisional Bilinear Diffie-Hellman (VDBDH) problem

Given $\left(P, a P, b P, c P, d P, c^{-1} P, d^{-1} P\right)$ unknown $a, b, c, d \in Z_{q}^{*}$, compute for $e(P, P)^{a b c^{-1}}$.

-Variants Computational Bilinear Diffie-Hellman (VCBDH) problem

Given $\quad\left(P, a P, b P, c P, d P, c^{-1} P, d^{-1} P\right) \quad$ with unknown $a, b, c, d \in Z_{q}^{*}$ compute for $e(P, P)^{a b d^{-1}}$.

- Discrete Logarithm Problem (DLP)

Given a cyclic additive group $G$ and generator $P$, the DLP is defined that given $(P, a P)$, compute $a \in Z_{q}^{*}$.

According to the existing schemes $[15,16]$, it is easy to known that both VDBDHP and VCBDHP are hard. Obviously, Discrete Logarithm Problem (DLP) is hard.

\section{Semantic security and security model}

\subsection{Semantic security}

A CLC $\rightarrow$ IBC heterogeneous signcryption scheme generally includes the following algorithm:

- Setup. Choose a security parameter $l$ to input. The PKG and KGC generate their own master key, and outputs the system's parameters params .

- CLPKC-KG. The algorithm runs by the KGC of the CLPKC system. Submit the identity $I D_{U}$ and a secret value $x_{U}$, the corresponding $s k_{s}$ is generated as the private key of the CLPKC user.
- IDPKC-KG. The algorithm runs by the PKG of the IDPKC system. By inputting a user's identity $I D_{U}$, the corresponding private key $S_{U}$ is generated.

- Signcryption. Given the sender's identity $I D_{s}$, the receiver's identity $I D_{r}$, the private key $S_{U}$ and the message $\mathrm{m}$, the sender runs the signcryption algorithm to generate a ciphertext $\sigma$.

- Unsigncryption. Given the sender's identity $I D_{s}$, the private key $S_{U}$ of the receiver and a ciphertext $\sigma$, the receiver computes and outputs the plaintext $m$, or the symbol $\perp$.

\subsection{Security Models}

\subsubsection{Confidentiality}

A heterogeneous signcryption scheme has the IND-CCA2 property if no probabilistic polynomial time adversary $A$ has a non-negligible advantage in the following game:

Setup. Challenger $C$ runs the setup algorithm with a security parameter $l$ and send system parameters and public keys to the $A$, while the master key is kept secret.

Phase 1. $A$ can ask several kinds of queries to the following random oracles.

- Key generation query. $A$ submits an identity $I D_{U}, C$ runs the IDPKC-KG algorithm to generate the private key $S_{U}$ and send it to $A$.

- Unsigncryption query. $A$ submits a signcrypted message $\sigma$ and an identity $I D_{s} . C$ computes the private key $S_{U}$. If $\sigma$ is a valid ciphertext, $C$ returns a message $m=$ Unsigncrypt $\left(\sigma\right.$, params, $\left.S_{U}\right)$; otherwise, an error symbol $\perp$ is returned.

Challenge. $A$ decides when Phase 1 ends. $A$ selects two plaintexts $m_{0}$ and $m_{1}$ of the same length, a sender's identity $I D_{A}$, and a receiver's identity $I D_{B}^{*}$, which it wants to challenge. $A$ is not allowed to ask for the private key to $I D_{B}$ in the first stage. $C$ then selects $b \in\{0,1\}$ and runs the corresponding algorithms to obtain the ciphertext $\sigma . \sigma$ is transmitted to $A$.

Phase 2. $A$ can perform the queries similar to those in Phase 1. $A$ cannot query the key extraction for the target identities. $A$ should not query the Unsigncryption for $\sigma$.

Guess. Finally, $A$ produces a bit $b^{\prime}, A$ wins the game if $b^{\prime}=b$.

The advantage of $A$ is defined as $\operatorname{Adv}(A)=\left|\operatorname{Pr}\left[\mathrm{b}^{\prime}=\mathrm{b}\right]-1\right|$, where $\operatorname{Pr}\left[\mathrm{b}^{\prime}=\mathrm{b}\right] \quad$ denotes the probability that $b^{\prime}=b$.

\subsubsection{Unforgeability I}

A CLPKC $\rightarrow$ IDPKC heterogeneous signcryption scheme is referred to as EUF-CMA-I . The game is defined between a challenger $C$ and an adversary $F_{I}$. If no 
probabilistic polynomial time adversary $F_{I}$ has a nonnegligible advantage against a challenger in the following game:

Setup. The challenger $C$ runs the setup algorithm. It sends public parameters params to the adversary $F_{I}$, while the master keys are kept secret. When $F_{I}$ receives params, $F_{I}$ outputs a target identity $I D^{*}$.

Attack. $F_{I}$ issues several kind of queries.

- Extract partial private key query. $F_{I}$ selects an identity $I D_{i}, C$ computes the partial private key $S_{i}$, and transmits it to $F_{I}$.

- Extract secret key query. $F_{I}$ produces an identity $I D_{i}, C$ computes the se cret key $x_{i}$, and transmits it to $F_{I}$.

- Request public key query. When $F_{I}$ receives a public key extraction query for an identity $I D_{i}, C$ runs the CLPKC-KG algorithm to the public key $p k_{i}$, and transmits it to $F_{I}$.

- Replace public key query. For any identity $I D_{i}$ and a valid public key $p k_{i}{ }^{\prime}, C$ replaces $p k_{i}$ with a value $p k_{i}{ }^{\prime}$.

- Signcrypt query. $F_{I}$ produces a message $m$, a sender's identity $I D_{s}$, and a re ceiver's identity $I D_{r}, C$ first finds the sender's private key $s k_{s}$ from the CLPKC$\mathrm{KG}$ algorithm. $C$ then runs signcryption algorithm and sends $\sigma$ to $F_{I}$.

Forgery. $F_{I}$ produces a tuple $\left(I D_{s}^{*}, \sigma^{*}, I D_{r}^{*}\right) \cdot I D_{s}^{*}$ cannot be queried a partial private key extraction. $F_{I}$ wins if the unsigncryption returns $m$.

The advantage of $F_{I}$ is defined as the probability that $F_{I}$ wins the EUF-CMA-I game.

\subsubsection{Unforgeability II}

A CLPKC $\rightarrow$ IDPKC heterogeneous signcryption scheme is referred to as EUF-CMA-II , The game is defined between a challenger $C$ and an adversary $F_{I I}$. If no probabilistic polynomial time adversary $F_{I I}$ has a nonnegligible advantage against a challenger in the following game:

Setup. The challenger $C$ runs the setup algorithm. It generates master secret keys $s_{K G C}, s_{P K G}$ and system's public parameters params to the adversary $F_{I I}$ while $s_{P K G}$ is kept secret. When $C$ receives the public parameters, $F_{I I}$ outputs a challenge identity $I D^{*}$.

- Extract secret key query. $F_{I I}$ produces an identity $I D_{i}, C$ computes the secret key $x_{i}$ from the CLPKC$\mathrm{KG}$ algorithm, and transmits it to $F_{I I}$.

- Request public key query. When $F_{I I}$ receives a public key extraction query for an identity $I D_{i}, C$ runs the CLPKC-KG algorithm to the public key $p k_{i}$, and transmits it to $F_{I I}$.

- Signcrypt query. $F_{I I}$ produces a message $m$, a sender's identity $I D_{s}$ and a re ceiver's identity $I D_{r} . C$ first finds the sender's private key $s k_{s}$ from the CLPKC$\mathrm{KG}$ algorithm. $C$ then runs the signcryption algorithm and sends $\sigma$ to $F_{I I}$.

Forgery. $F_{I I}$ produces a tuple $\left(I D_{s}^{*}, \sigma^{*}, I D_{r}^{*}\right) . I D_{s}^{*}$ cannot be queried a partial private key extraction. $F_{I I}$ wins if the unsigncryption returns $m . \sigma$ was not produced by any signcrypt query.

The advantage of $F_{I I}$ is defined as the probability that $F_{I I}$ wins the EUF-CMA-II game.

\section{Describe the scheme}

The heterogeneous signcryption scheme is described in this section. It can be allowed as the sender in the CLPKC system and as the receiver in the IDPKC system. The proposed scheme is described as follows.

Setup. In the heterogeneous system, let $G_{1}$ be a cyclic additive group of prime order $q$ and $G_{2}$ be a cyclic multiplicative group of the same order. Let $P$ be the generator of $G_{1}$. A bilinear map is defined as $e: G_{1} \times G_{1} \rightarrow G_{2}$. Three cryptographic hash functions are chosen,namely, $H_{1}: 0,1^{*} \rightarrow G_{1}, H_{2}: G_{2} \rightarrow 0,1^{l_{m}}$ and $H_{3}: 0,1^{*} \rightarrow Z_{\mathrm{q}}^{*}$. where $l_{m}$ is the length of a message. The KGC selects $s_{1} \in Z_{\mathrm{q}}^{*}$ as the master secret key and sets $P_{1}=s_{1} P$ as the public key of the CLPKC system . The PKG selects $s_{2} \in Z_{\mathrm{q}}^{*}$ as the master secret key and sets $P_{2}=s_{2} P$ as the public key of the IDPKC system . The global public parameters are param $=\left\langle G_{1}, G_{2}, e, P, P_{1}, P_{2}, H_{1}, H_{2}, H_{3}\right\rangle$ and the master secret key are $s_{1}$ and $s_{2}$.

CLPKC-KG. As a sender in the CLPKC system, it uses an identity $I D_{A}$ and com putes the private key using the following algorithm.

- Compute $Q_{A}=H_{1}\left(I D_{A}\right)$ and set the partial private key $S_{A}=s_{1}^{-1} Q_{A}$.

- Choose a random secret value $x_{A} \in Z_{q}^{*}$.

- Compute the public key $p k_{A}=x_{A} P$ and the private key $D_{A}=x_{A} S_{A}$.

IDPKC-KG. The PKG computes the private key of an identity $I D_{B}$ as $S_{B}=S_{2}^{-1} Q_{B}$ where $Q_{B}=H_{1}\left(I D_{B}\right)$.

Signcryption. The system parameters, namely, the message $\mathrm{m}$ of length $l_{m}$, the receiver's identities $I D_{B}$ are given, Then the sender uses the following algorithm:

- Choose a random number $r \in Z_{q}^{*}$ and compute $U_{1}=r P_{2}, U_{2}=r Q_{A}$. 
- Compute $R=e\left(P, Q_{B}\right)^{r}$ and $k=H_{2}(R)$.

- Compute $c=m \oplus R, h=H_{3}\left(m, k, U_{1}, U_{2}\right), V=(r+h) D_{A}$.

- Output ciphertext as $\sigma=\left(c, U_{1}, U_{2}, V\right)$.

Unsigncryption. When the system receives a ciphertext $\sigma=\left(c, U_{1}, U_{2}, V\right)$ and the system parameters, the receiver with an identity $I D_{B}$ unsigncrypts the ciphertext $\sigma$ as follows:

- Compute $R=e\left(U_{1}, S_{B}\right)$ and $k=H_{2}(R)$.

- Recover $m=c \oplus R$.

- Compute $h=H_{3}\left(m, k, U_{1}, U_{2}\right)$.

-Accept the message if and only if the equation holds: $e\left(P_{1}, V\right)=e\left(p k_{A}, U_{2}+h Q_{A}\right)$

Correctness. The following equations show the correctness of the scheme.

When receiving the ciphertext, the receiver can decrypt the ciphertext as follows:

$$
R=e\left(U_{1}, S_{B}\right)=e\left(r P_{2}, s_{2}^{-1} Q_{B}\right)=e\left(P, Q_{B}\right)^{r}
$$

Simultaneously, the receiver can verify the following equalities to show:

$$
e\left(P_{1}, V\right)=e\left(s_{1} P,(r+h) D_{A}\right)=e\left(s_{1} P,(r+h) x_{A} S_{A}\right)=
$$
$e\left(x_{A} P,(r+h) S_{1} S_{A}\right)=e\left(p k_{A},(r+h) Q_{A}\right)=e\left(p k_{A}, r Q_{A}+h Q_{A}\right)=$ $e\left(p k_{A}, U_{2}+h Q_{A}\right)$

The scheme can be modified into environments with different systems parameters. For example, the PKG has system parameters $\left\langle G_{1}^{*}, G_{2}^{*}, P^{*}, H_{1}, H_{2}, H_{3}\right\rangle$ and the $\mathrm{KGC}$ has the system parameters $\left\langle G_{1}, G_{2}, P, H_{1}, H_{2}, H_{3}\right\rangle$.The scheme will remain secure.

\section{Security Analysis}

This section will show the scheme's security analysis, including confidentiality and unforgeability.

\subsection{Confidentiality}

Theorem 1. In the random oracle, if an IND-CCA2 adversary $C$ can attack our proposed scheme, then there exists an algorithm $C$ that can solves the VDBDH problem.

Proof. The distinguisher $C$ inputs $\left(P, a P, b P, c P, d P, c^{-1} P, d^{-1} P, \theta\right)$ and attempts to decide whether $\theta=e(P, P)^{a b c^{-1}}$ or not. $C$ will run $A$ as a subroutine and act as $A^{\prime} s$ challenger in the IND-CCA2 game. We model $H_{i}(i=1,2,3)$ as random oracles. $C$ keeps three lists $L_{1}, L_{2}, L_{3}$ to store the answers.

Setup. At the beginning of the game, $C$ runs the setup algorithm and set $P_{b}=c P$ and provides the system parameters to attacker $A$.

Phase 1. $A$ can request several queries. To respond to these queries, $\mathrm{H}_{1}, \mathrm{H}_{2}, \mathrm{H}_{3}$ are random oracles controlled by $C$ as follows.

- $H_{1}$-query: $C$ selects a random number $j \in\left\{1,2, \cdots, q H_{1}\right\} . A$ asks for a polynomial bounded number of $H_{1}$ queries on identities of his choice. At the $j$-th $H_{1}$ query, $C$ answers by $H_{1}\left(I D_{j}\right)=b P$. For queries $H_{1}\left(I D_{i}\right)$ with $i \neq j, C$ selects $e_{i} \in Z_{q}^{*}$, puts the pair $\left(I D_{i}, e_{i}\right)$ in list $L_{1}$ and answers $H_{1}\left(I D_{i}\right)=e_{i} P$.

- $H_{2}, H_{3}$-queries. When $A$ asks queries on these hash values, $C$ checks the list. If an entry for the query is found, it will be return to $A$. Otherwise, a random value is chosen to send to $A$, and the answer will then be stored in the list.

- Key extraction query. When $A$ asks a key extraction query in identity $I D_{i}$, if $I D_{i}=I D_{j}$, then $C$ fails and stops. If $I D_{i} \neq I D_{j}, C$ finds $\left(I D_{i}, e_{i}\right)$ from $H_{1}$ (which indicates that $C$ previously answered $H_{1}\left(I D_{i}\right)=e_{i} P$ on an $H_{1}$ query on $\left.I D_{i}\right) . C$ updates the list, and then computes the corresponding private key $e_{i} c^{-1} P$. C returns it to $A$.

-Unsigncryption queries. For an unsigncryption query in a ciphertext $\sigma^{\prime}$ for iden tities $I D_{A}$ and $I D_{B} . C$ follows the steps below:

Case 1: $I D_{B}=I D_{j} . C$ always answers $A$ that $\sigma^{\prime}$ is invalid. If $\sigma^{\prime}$ is valid from $A^{\prime} s$ viewpoint, $C$ will fail.

Case 2: $I D_{B} \neq I D_{j} . C$ computes $R^{\prime}=e\left(U_{1}^{\prime}, S_{B}\right)(C$ could obtain $S_{B}$ from the key extraction algorithm because $\left.I D_{B} \neq I D_{j}\right) . C$ then runs the $H_{2}$ simulation algorithm to obtain $k^{\prime}=H_{2}\left(R^{\prime}\right)$ and computes $m^{\prime}=c \oplus R^{\prime}$.

Finally, $C$ runs the $\mathrm{H}_{3}$ simulation algorithm to generate $\quad h^{\prime}=H_{3}\left(m^{\prime}, k^{\prime}, U_{1}^{\prime}, U_{2}^{\prime}\right)$ and checks if $e\left(P_{1}, V^{\prime}\right)=e\left(p k_{A}, U_{2}^{\prime}+h^{\prime} Q_{A}\right)$ holds. If the above equation does not hold, $C$ returns $\perp$. Otherwise, $C$ returns $m^{\prime}$.

Challenge. $A$ outputs two plaintexts $m_{0}, m_{1}$ to $C$. $C$ randomly chooses $b \in\{0,1\}$ and computes the signcryption of message $m_{b}$ as follows.

- It sets $U_{1}^{*}=a P$, obtains $k^{*}=H_{2}(\theta)$ (where $\theta$ is

$C$ candidate for the VDBDHP ).

- Compute $c_{b}=m_{b} \oplus R$

- Randomly choose $x^{*}, h^{*} \in Z_{q}^{*}$.

- Compute $U_{2}^{*}=-h^{*} Q_{A}+x^{*} p k_{A}$ and $V^{*}=x^{*} P$.

- Adds $\left(c^{*}, U_{1}^{*}, U_{2}^{*}, V^{*}\right)$ to list $L_{3}$.

Finally, $C$ produces the challenged ciphertext $\sigma^{*}=\left(\mathrm{c}_{b}, \mathrm{U}_{1}^{*}, \mathrm{U}_{2}^{*}, \mathrm{~V}^{*}\right)$ and send it to $A$.

Phase 2. $A$ performs new queries that are treated the same way as in Phase 1.

Guess. $A$ produces a bit $b^{\prime}$ as its guess. At the moment, if $b=b^{\prime} \quad, \quad C \quad$ outputs $\theta=e\left(U_{1}^{*}, S_{I D_{j}}\right)=e\left(a P, c^{-1} b P\right)=e(P, P)^{a b c^{-1}}$ as a solution of the VDBDHP. Otherwise, $C$ stops and outputs "failure". 


\subsection{Unforgeability I}

Theorem 2. In the random oracle model, if an EUF-CMA-I adversary $F_{I}$ can attack our proposed scheme, then there exists an algorithm $C$ that can solve the VCBDH problem.

Proof. If an EUF-CMA-I adversary $F_{I}$ exists, then we construct a simulator $C$ that uses $F_{I}$ to compute $e(P, P)^{a b d^{-1}}$ from an instance $\left(P, a P, b P, c P, d P, c^{-1} P, d^{-1} P\right)$ of the VCBDHP.

Setup. The challenger $C$ runs the setup algorithm. It sets $P_{1}=d P$, and sends the system parameters params $=\left\{G_{1}, G_{2}, e, P_{1}, P_{2}, H_{1}, H_{2}, H_{3}\right\}$ to $F_{I}$, then $F_{I}$ outputs a challenge identity $I D^{*}$.

- $H_{1}$-query. When $C$ receives a query $\left(I D_{j}, \alpha_{j}, H_{1}\left(I D_{j}\right), D_{j}\right)$. If $\left(I D_{j}, \alpha_{j}, H_{1}\left(I D_{j}\right), D_{j}\right)$ exists in $L_{1}$, then $h_{j}$ is returned to $F_{I}$. Otherwise, $C$ does as follows:

(1) If $I D_{j} \neq I D^{*}$, select $\alpha_{j} \in Z_{q}^{*}$ at random, then compute $H_{1}\left(I D_{j}\right)=\alpha_{j} P, D_{j}=\alpha_{j} P_{1}$;otherwise, set $\alpha_{j}=D_{j}=\perp, H_{1}\left(I D_{j}\right)=b P$.

(2) Put $\left(I D_{j}, \alpha_{j}, H_{1}\left(I D_{j}\right), D_{j}\right)$ into $L_{1}$, return $H_{1}\left(I D_{j}\right)$.

- $H_{i}(i=2,3)$-query. When $C$ receives a query, if the corresponding query exists in $L_{i}(i=2,3), C$ returns it to $F_{I}$. Otherwise, $C$ randomly chooses an number as the query answer and return to $F_{I}$. Meanwhile, $C$ puts the query result into $L_{i}$.

- Public key query. When $C$ receives a query $\left(I D_{j}, x_{j}, p k_{j}\right)$. If $\left(I D_{j}, x_{j}, p k_{j}\right)$ exists in the public keylist, then $p k_{j}$ is returned to $F_{I}$, Otherwise, $C$ chooses $x_{j} \in Z_{q}^{*}$ at random, computes $p k_{j}=x_{j} P$, then puts $\left(I D_{j}, x_{j}, p k_{j}\right)$ into the public key-list and return $p k_{j}$ as answer.

- Extract secret key query. When $C$ receives a query $I D_{j}$. If $C$ replaces the public key of $I D_{j}$, then it returns $\perp$. Otherwise, $\left(I D_{j}, x_{j}, p k_{j}\right)$ exists in the keylist, and $C$ returns $x_{j}$ as answer.

- Extract partial private key query. When $C$ receives a query $\left(I D_{j}, x_{j}, D_{j}\right), C$ does as follows:

(1) If $I D_{j}=I D^{*}$,then $C$ aborts; if $\left(I D_{j}, \alpha_{j}, H_{1}\left(I D_{j}\right), D_{j}\right)$ exists in $L_{1}, D_{j}$ is returned to $F_{I}$; otherwise, $C$ first makes a query to $H_{1}$.

(2) Put $\left(I D_{j}, x_{j}, D_{j}\right)$ into $L_{1}$; return $D_{j}$.

- Replace public key query. When $C$ receives a replace public key query $\left(I D_{j}, p k_{j}^{\prime}\right), C$ first finds $\left(I D_{j}, x_{j}, p k_{j}\right)$ on the public key-list, then $C$ updates the public key-list with tuple $\left(I D_{j}, \perp, p k_{j}^{\prime}\right)$, and sets $x_{j}=\perp, p k_{j}=p k_{j}^{\prime}$.
- Signcryption query. When $C$ receives a signcryption query, let $I D_{A}, I D_{B}$ be the identies of the sender and receiver, respectively, $m$ be the plaintext, $C$ will do as follows:

(1) If $I D_{A} \neq I D_{j} . C$ computes the private key $S_{I D_{A}}$ corresponding to $I D_{A}$ by running the key extraction query algorithm. Then $C$ answers the query.

(2) If $I D_{A}=I D_{j}, C$ simulates the signcryption algorithm to create a signcryption in the following steps. $C$ chooses $x, h \in Z_{q}^{*}$ randomly, and computes $U_{1}=x P_{b}, R=e\left(U_{1}, S_{B}\right)$ ( $C$ could obtain $S_{B}$ from the key extraction algorithm because $\left.I D_{B} \neq I D_{j}\right)$. C runs the $H_{2}$ simulation algorithm to find $k=H_{2}(R)$ and computes $c=m \oplus R$. Then $C$ computes $U_{2}=-h Q_{A}+x p k_{A}$ and $V=x P$, and adds $\left(c, U_{1}, U_{2}, h\right)$ to list $L_{3}$. If a collision happens, $C$ outputs fail and exits. Otherwise, $C$ gives the ciphertext $\sigma$ to $F_{I}$.

Attack. $F_{I}$ adaptively performs a polynomially bounded number of queries to the various oracles in this phase.

Forge. Eventually, $F_{I}$ outputs a tuple $\left(m^{*}, \sigma^{*}, I D_{s}^{*}, p k_{s}^{*}\right)$. If $I D_{s}^{*} \neq I D^{*}, C$ aborts. Otherwise, by forking lemma, $C$ chooses different $h, h^{*}$ and interacts with $F_{I}$ the same random tape, then the adversary can give a different forger $\sigma^{*} \cdot \sigma^{*}$ and $\sigma$ should verified by the equation $e\left(P_{1}, V\right)=e\left(p k_{A}, U_{2}+h Q_{A}\right)$ and $e\left(P_{1}, V^{*}\right)=e\left(p k_{A}, U_{2}+h^{*} Q_{A}\right)$. Where if $P_{1}=d P$ and $p k_{A}=\beta P, C$ can compute $b d^{-1} P=\frac{V-V^{*}}{\beta\left(h-h^{*}\right)}$, Simultaneously, $C$ delivers the value of $e(P, P)^{a b d^{-1}}$ as $e\left(a P, b d^{-1} P\right)$. Hence, $C$ successfully solves the VCBDHP.

\subsection{Unforgeability II}

Theorem 3. In the random oracle model, if an EUF-CMA-II adversary $F_{I I}$ can attack our proposed, then there exists an algorithm $C$ that can solve the Discrete Logarithm Problem (DLP).

Proof. If an EUF-CMA-II adversary $F_{I I}$ exists, then we construct a simulator $C$ that uses $F_{I I}$ to compute $a \in Z_{q}^{*}$ from an instance $\langle P, a P\rangle$ of the DLP.

Setup. $C$ first runs the setup algorithm to generate $s_{1}, s_{2}$ as the master keys, and sets $P_{2}=s_{2} P$, then gives $s_{1}$, params to the attacker $F_{I I}, s_{2}$ is kept secret. $F_{I I}$ outputs a challenge identity $I D^{*}$.

- $H_{i}(1,2,3)$-query. When $C$ receives a query, if the corresponding query exists in $H_{i}$-list, $C$ returns it to $F_{I I}$. Otherwise, $C$ randomly chooses an integer as the query result and returns it to $F_{I I}$. Meanwhile, $C$ puts the query result into the $H_{i}$-list. 
- Public Key query. When $C$ receives a query $\left(I D_{j}, x_{j}, p k_{j}\right)$. If $\left(I D_{j}, x_{j}, p k_{j}\right)$ exists in the public key-list, then $C$ returns $p k_{j}$. Otherwise, $C$ chooses $x_{j} \in Z_{q}^{*}$ at random, computes $p k_{j}=x_{j} P$, then puts $\left(I D_{j}, x_{j}, p k_{j}\right)$ into the public key-list and returns $p k_{j}$ as answer.

- Signcryption query. The same as Unforgeability I's Signcryption query.

Attack. $F_{I I}$ adaptively performs a polynomially bounded number of queries to the various oracles in this phase.

Forge. Eventually, $F_{I I}$ outputs a tuple $\left(m^{*}, \sigma^{*}, I D_{s}^{*}, p k_{s}^{*}\right)$. If $I D_{s}^{*} \neq I D^{*}, C$ aborts; otherwise, $C$ randomly chooses $h \in Z_{q}^{*}$, then the adversary can give a forger $\sigma$. We know that $\sigma$ should verified by the equation $e\left(P_{1}, V^{*}\right)=e\left(p k_{A}^{*}, U_{2}^{*}+h Q_{A}^{*}\right)$. And $C$ set $p k_{A}^{*}=a P$ and compute the equation, then $C$ obtain the result $a=\frac{s_{1} V^{*}}{U_{2}^{*}+h Q_{A}^{*}}$ as the solution to given DLP. Hence, $C$ successfully solves the DLP.

\section{Performance analysis}

We compare the proposed scheme with other existing signcryption schemes [2],[7],[18]. The Table 1 shows the efficiency and distinction of the schemes. We denote $\mathrm{Pa}$ as the total number of pairing computations required, mark the $\mathrm{Mu}$ as the total number of point multiplications required and denote the Ex as the total number of exponentiations required. We compare our scheme with scheme [2],[7],[18] even though these schemes are all in the same cryptosystems. The results are shown in Table 1 and Table 2. As shown in Table 1 and Table 2, our scheme has a lower computation cost than other schemes.

Table 1. Comparison with existing scheme for signcryption.

\begin{tabular}{|c|c|c|c|c|}
\hline Scheme & $\mathrm{Pa}$ & $\mathrm{Mu}$ & $\mathrm{Ex}$ & Master key \\
\hline $\mathrm{Sun}[2]$ & 1 & 3 & 1 & Same \\
\hline $\operatorname{Li}[7]$ & 2 & 3 & 2 & Same \\
\hline $\mathrm{F} \mathrm{Li}[18]$ & 1 & 3 & 1 & Same \\
\hline $\begin{array}{c}\text { Our } \\
\text { scheme }\end{array}$ & 1 & 2 & 1 & Different \\
\hline
\end{tabular}

Table 2. Comparison with existing scheme for unsigncryption.

\begin{tabular}{|c|c|c|c|c|}
\hline Scheme & $\mathrm{Pa}$ & $\mathrm{Mu}$ & $\mathrm{Ex}$ & Master key \\
\hline Sun[2] & 4 & 1 & 0 & Same \\
\hline $\mathrm{Li}[7]$ & 3 & 2 & 2 & Same \\
\hline
\end{tabular}

\begin{tabular}{|c|c|c|c|c|}
\hline F Li[18] & 5 & 0 & 0 & Same \\
\hline $\begin{array}{c}\text { Our } \\
\text { scheme }\end{array}$ & 3 & 1 & 0 & Different \\
\hline
\end{tabular}

In the simulation, we show the time by measuring the performance of the Signcryption and Unsigncryption. Our program is written in with the PBC library [17], and we run on a personal computer with $3.10 \mathrm{GHz} \mathrm{CPU}$ and 2GB of RAM, using Linux operating system. In order to the effect of comparison, we will adopt Y Li et al [11]. Both schemes are set up in heterogeneous system, and both the schemes use different system parameters. We can get the result as Fig 1, which SC means signcryption operation and USC means unsigncryption operation. In the performance of the Signcryption and Unsigncryption, our scheme has been improved in terms of efficiency.

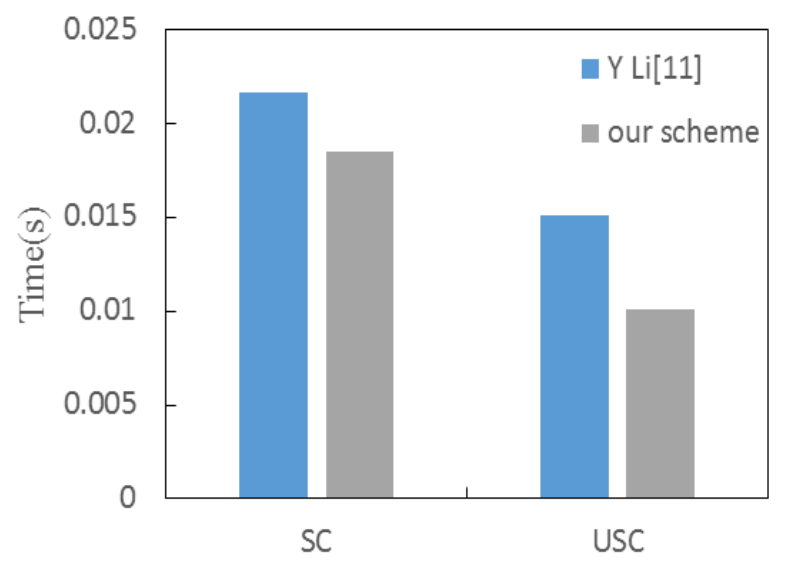

Fig. 1. Performance comparison between Li's and our schemes.

\section{Conclusion}

We have developed an efficient signcryption scheme for the CLPKC $\rightarrow$ IDPKC heterogeneous system. Compared with the existing signcryption schemes, the proposed scheme chooses different master secret keys in different systems. The security models are provided, and the proposed scheme ensures the confidentiality and unforgeability of data in the random oracle model. The proposed is more secure because it uses different master keys. Furthermore, the proposed scheme can be used in environments with different system parameters.

\section{Acknowledgements}

The work was supported by the National Natural Science Foundation of China under grant 61562077, 61462077, 61662071, 61662069, and Natural Science Foundation of Gansu Province for Distinguished Young Scholars 1308RJDA007. 


\section{References}

1. Zheng Y. Digital signcryption or how to achieve cost in AdvancesinCryptology'97 on Springer, 1997. pp.165-179.

2. Sun, Li H.Efficient signcryption between TPKC and IDPKC and its multi-receiver construction, Science China Information Sciences, Vol 53, 2010, pp.557566.

3. Shamir. Identity-Based cryptosystems and signature schemes, Springer, Heidelberg, 1985, pp.47-53.

4. An J H, Dodis Y, and Rabin T. On the Security of Joint Signature and Encryption, Springer, Berlin, 2002, pp.83-107.

5. Huang Q, Wong D S, and Yang G. Heterogeneous signcryption with key Privacy, Computer Journal, Vol 54, 2011, pp.525-536.

6. Fu, Li, and Liu W. IDPKC-to-TPKC construction of multi-receiver signcryption, Proceedings of the INCoS (5) on IEEE, 2013, pp.335- 339.

7. Li, Zhang $\mathrm{H}$, and Takagi $\mathrm{T}$. Efficient signcryption for heterogeneous systems, IEEE Systems Journal, Vol 7, 2013, pp.420-429.

8. Zhang et al, CLPKC-to-TPKI Heterogeneous Signcryption Scheme with Anonymity, Acta Electronica Sinica, Vol 44, 2016, pp.2432-2439.

9. Al-Riyami S S, and Paterson K G. Certificateless public key cryptography, Advances in CryptologyAsiacrypt on Springer, 2003, pp.452-473.

10. Zhou Y W, Yang B, and Zhang W Z. Provably secure and efficient certificateless generalized signcryption, Chinese Journal of Computers, Vol 39, 2016, pp.543-551.

11. Li Y, Wang C, Zhang Y, and Niu S. Privacy preserving multi - receiver signcryption scheme for heterogeneous systems, Security \& Communication Networks, Vol 9, 2017, pp.4574-4584.

12. Li F, Shirase M, and Takagi T. Efficient Multi-PKG ID-Based Signcryption for Ad Hoc Networks, Information Security and Cryptology on Springer, 2009, pp.289-304.

13. Al-Riyami, S. S, Paterson, and K. G. Certificateless public key cryptography. International Conference on the Theory and Application of Cryptology, Springer, Berlin, 2003, pp.452-473.

14. Bellare, M. and Rogaway P. Random Oracles are Practical, 1993, pp.366-368.

15. Bao F, Deng R H, and Zhu H F. Variations of Diffie-Hellman Problem, in Information and Communications Security, Huhehaote, China, 2003, pp.301-312.

16. Chow S S M, Yiu S M, and Hui L C K. Efficient Forward and Provably Secure ID-Based Signcryption Scheme with Public Verifiability and Public Ciphertext Authenticity, Vol 2971, 2004, pp.352-369.

17. PBC Library. http://crypto.stanford.edu/pbc/.
18. Li, Fagen, Y. Han, and C. Jin. Practical access control for sensor networks in the context of the Internet of Things , Computer Communications, Vol 89,2016, pp.154-164. 